\title{
Video Article \\ A Conditioned Place Preference Protocol for Measuring Incubation of Craving in Rats
}

\author{
Yongmei Sun ${ }^{1}$, Gaowei Chen ${ }^{1,2}$, Kuikui Zhou ${ }^{1}$, Yingjie Zhu ${ }^{1}$ \\ ${ }^{1}$ Brain Cognition and Brain Disease Institute, Shenzhen Institutes of Advanced Technology, Chinese Academy of Sciences \\ ${ }^{2}$ Grain College, Henan University of Technology
}

Correspondence to: Yongmei Sun at ym.sun1@siat.ac.cn, Yingjie Zhu at yj.zhu1@siat.ac.cn

URL: https://www.jove.com/video/58384

DOI: doi: $10.3791 / 58384$

Keywords: Behavior, Issue 141, Morphine, Balanced biased design, Conditioned place preference, Incubation of craving, Long-term reward memory, Somatosensory cues

Date Published: 11/6/2018

Citation: Sun, Y., Chen, G., Zhou, K., Zhu, Y. A Conditioned Place Preference Protocol for Measuring Incubation of Craving in Rats. J. Vis. Exp. (141), e58384, doi:10.3791/58384 (2018).

\section{Abstract}

A major cause of repeated relapses is a craving for the drug. Drug craving increases progressively during the abstinence period, a phenomenon termed incubation of drug craving. Here, we describe a morphine conditioned place preference (CPP) protocol for measuring the incubation of craving in rats. In this protocol, a CPP paradigm mainly employing somatosensory cues is used to establish a long-term reward memory of morphine. A three-chamber CPP box that differs in the texture of the chamber floor is constructed. First, the animals are tested for their baseline preference to the two side chambers for three consecutive days. Then, they are injected intraperitoneally with morphine/saline and put into their non-preferred/preferred chamber for $45 \mathrm{~min}$. After 6 days of conditioning, their preference to the side chambers is tested for $15 \mathrm{~min}$ at different time points after the last conditioning session. With this paradigm, the reward memory of morphine could last for at least 18 days. To test whether the above-mentioned protocol can model increased craving, the number of entrances into the two side chambers are counted during the abstinence period. The results show that the entrances increased, suggesting that the CPP paradigm could mimic the incubation of craving Future studies can employ this model to study neural mechanisms underlying long-term memory and incubation of craving.

\section{Video Link}

The video component of this article can be found at https://www.jove.com/video/58384/

\section{Introduction}

Drug addiction is a chronic disease ${ }^{1}$ with several different stages ${ }^{2}$ : binge/intoxication, withdrawal/negative affect, and preoccupation/anticipation. In the initial binge/intoxication stage of the drug addiction cycle, subjects take drugs due to the reinforcing effect ${ }^{2}$. In the withdrawal/negative affect stage, subjects experience negative somatic and emotional states of drug withdrawal, which promote negative reinforcement mechanisms associated with the development of addiction ${ }^{3}$. In the preoccupation/anticipation stage, subjects reinstate drug-seeking after extinction, which could be triggered by drug-associated cues or stressors. In animal studies of drug addiction, conditioned place preference (CPP) paradigms are used to mimic the positive reinforcing effect of drugs ${ }^{4,5,6}$ and, thus, it is used to model the initial stage of the drug addiction cycle. Morphine, a member of the opioid family, produces a positive reinforcing effect by activating the opioid receptors in the brain ${ }^{7,8}$.

The CPP paradigm is based on the principle of classical (Pavlovian) conditioning and commonly used to measure the rewarding effect of drugs of abuse, such as morphine ${ }^{4,5,9,10}$, due to its easy availability across laboratories. A typical CPP paradigm consists of three phases: preconditioning, conditioning and test phases. During the conditioning phase, the rewarding properties of a drug (unconditioned stimulus) are paired with one or more neutral contextual stimuli, which gain rewarding properties after several conditioning pairings and serve as conditioned stimuli. During the test phase, the animals' preference for drug-paired compartments are measured and reflected as their reward memory. For this reason, the CPP paradigm is also used to investigate the mechanisms of learning and memory. To establish a long-term reward memory of morphine, variables that affect the establishment of CPP have been adjusted and optimized with the below described protocol ${ }^{11}$. Contextual stimuli are a combination of visual, tactile and odor cues. Variations of contextual stimuli and testing time points are made according to different research purposes. For example in our previous study ${ }^{12}$, the somatosensory cues (different floor textures) were mainly used as contextual stimuli to investigate the role of the caudal part of the insular cortex, a brain region with somatosensory function, in the acquisition and maintenance of long-term reward memory. The design of the apparatus and the assignment of rats to preferred/non-preferred sides affect the establishment of CPP. A three-chamber apparatus instead of a two-chamber apparatus is used in the below protocol to avoid forced choices. The balance of two side chambers and the initial assignment of animals into them affect the acquisition of CPP ${ }^{4,13}$. Thus, a balanced design of the side chambers and biased assignment of animals are applied in this protocol to acquire a strong place preference. Maintenance of long-term reward memory requires more conditioning trials during the conditioning phase. To reduce the time cost during conditioning phase, two conditioning trials per day are carried out and morphine/saline injections are counterbalanced to avoid the acute withdrawal effect of morphine in the saline paired trials ${ }^{4}$.

Reinstatement animal models ${ }^{14}$ have been established to model the preoccupation/anticipation stage of drug addiction cycle. CPP is also utilized to study reinstatement that models the relapse to addictive behavior ${ }^{5}$. Relapse is one of the barriers in the treatment of drug addiction. Craving 
is one cause of repeated relapse. The craving for drugs increases constantly during the abstinence period, a phenomenon termed incubation of craving ${ }^{15,16}$. Incubation of craving is widely studied in the drug self-administration paradigm but not the CPP paradigm. The use of the CPP paradigm to reflect incubation of craving was first proposed by Li et al. ${ }^{17}$. Their results ${ }^{17}$ showed that the CPP scores of low doses of morphine $(1 \mathrm{mg} / \mathrm{kg}$ and $3 \mathrm{mg} / \mathrm{kg})$ but not a high dose $(10 \mathrm{mg} / \mathrm{kg})$ increased progressively during the first 14 days of abstinence. Similarly, mice injected with a low dose of cocaine showed increased CPP scores over time ${ }^{18}$. The increased CPP scores of the low doses of morphine were used to reflect incubated craving ${ }^{17}$. Both duration spent in and number of entries into the drug-associated environment are suggested to reflect the reinforcing conditioned responses in the CPP paradigm ${ }^{19}$. Most of the recent studies use CPP scores to directly or indirectly reflect time spent in drugpaired chambers while the number of entries to side chambers as a parameter is often neglected. For these reasons, a morphine dose of $10 \mathrm{mg} /$ $\mathrm{kg}$ was used and the number of entries was measured in our study ${ }^{11}$. The results show that entrances of rats to the side chambers increased significantly during the abstinence period ${ }^{11}$. This indicates that both CPP scores and number of entries are useful parameters in assessing increased craving for drugs and the CPP paradigm is applicable to model the incubation of craving phenomenon. However, to reduce the number of animals required, a group of morphine-experienced animals is tested repeatedly; thus, the effect of extinction could not be excluded with the current within subject design. A between subject design with the same protocol can overcome this limitation.

The following describes the CPP protocol that established long-term reward memory and modeled increased craving. Briefly, after three preconditioning days, rats go through six conditioning days with two conditioning trials per day and then are tested at different time points after the last conditioning trial. In this CPP paradigm, a three-chamber polyvinyl chloride (PVC) box used as the CPP apparatus is constructed and the floor of the apparatus is balanced. The apparatus consists of two large black side chambers $(30 \mathrm{~cm} \times 25 \mathrm{~cm} \times 30 \mathrm{~cm}, \mathrm{~L} \times \mathrm{W} \times \mathrm{H})$ and one middle white chamber $(11 \mathrm{~cm} \times 25 \mathrm{~cm} \times 30 \mathrm{~cm}, \mathrm{~L} \times \mathrm{W} \times \mathrm{H})$ with white smooth PVC floor. The two side chambers have different floor textures as somatosensory cues: one with a grid plexiglass floor, the other with a rough PVC floor.

\section{Protocol}

The experimental procedures were performed in accordance with the guidelines for the National Care and Use of Animals and the experiments were approved by the Institutional Animal Care and Use Committee at Shenzhen Institutes of Advanced Technology, Chinese Academy of Sciences.

\section{Animal Habituation}

1. Obtain male adult rats from animal laboratory and maintain them under a $12 \mathrm{~h}$ light/dark cycle (light on from 7:00 to 19:00) with free access to food and water.

2. Handle the animals by playing with them or patting them twice per day for two weeks before the experiments.

3. Randomly assign animals into saline or morphine groups.

\section{Drug Preparation}

1. Prepare morphine hydrochloride as $10 \mathrm{mg} / \mathrm{mL}$ and inject rats intraperitoneally (i.p.) at a volume of $1 \mathrm{~mL} / \mathrm{kg}$.

2. As a control, inject rats with saline at a volume of $1 \mathrm{~mL} / \mathrm{kg}$.

\section{CPP Apparatus}

1. Construct a three-chamber CPP apparatus with different tactile (floor) cues and test rats' initial preference to two side chambers. The apparatus consists of two large black side chambers $(30 \mathrm{~cm} \times 25 \mathrm{~cm} \times 30 \mathrm{~cm}, \mathrm{~L} \times \mathrm{W} \times \mathrm{H})$ and one middle white chamber $(11 \mathrm{~cm} \times 25 \mathrm{~cm} \times$ $30 \mathrm{~cm}, \mathrm{~L} \times \mathrm{W} \times \mathrm{H}$ ) with white smooth PVC floor. The two side chambers have different floor textures as somatosensory cues: one with a grid plexiglass floor, the other with a rough PVC floor.

2. Vary the combination of tactile cues to make sure that the apparatus is unbiased, since a biased apparatus is susceptible to yielding false positive results ${ }^{4}$.

NOTE: An important methodological issue in the design of the CPP apparatus is whether the apparatus is "biased" or "unbiased" ${ }^{4}$. If one group of untrained rats shows no initial preference for one side chamber over another, the apparatus is considered "unbiased". Otherwise, consider it as "biased"13.

\section{CPP Procedure}

1. Establish the morphine-induced CPP paradigm mainly according to previous studies ${ }^{21,22}$ with modification of conditioning time points, number of conditioning sessions, conditioning trial period and testing time points ${ }^{20}$. A short description is given below, of which the experimental schedule is depicted in Figure 1.

2. Pre-conditioning phase

1. During this phase (day 1 to 3 ), remove guillotine doors to allow free access to the entire apparatus and place the animal in the middle chamber.

2. Record the activity of the animals for $15 \mathrm{~min}$.

3. Calculate the time spent in the two side chambers on day 3 as the baseline and refer this as TO.

4. Remove rats that enter less than -four times to either of the side chambers from the experiment ${ }^{23}$ as these rats are likely to have problems in general well-being (e.g., dirty fur and having tumors). Check the health state of the animals during the whole experimental period.

3. Conditioning phase

NOTE: Conditioning phase includes 6 days (day 4 to 9 ). 
1. For morphine-conditioned subjects, inject rats i.p. with morphine $(10 \mathrm{mg} / \mathrm{kg})$ or saline $(1 \mathrm{~mL} / \mathrm{kg})$ alternately in the morning $(9: 00)$ and evening (19:00) sessions (10 h apart).

2. Confine rats immediately after each injection to its drug-paired (non-preferred) or saline-paired (preferred) chamber for 45 min (Figure 1B).

3. Keep the video recording system on.

NOTE: According to our experience, around $5 \%$ of rats have the respiratory depression after the first injection of morphine (10 mg/kg). In such a case, to increase the survival rate of respiratory depressed rats, get the rat out of the conditioning chamber, pull its tongue out and put it on a white tissue paper in its home cage.

4. For saline-conditioned controls, give rats saline injections $(1 \mathrm{~mL} / \mathrm{kg})$ prior to exposure to one side chamber of the CPP apparatus during the morning session and to the other side during the evening session for $45 \mathrm{~min} / \mathrm{session}$ in line with morphine group (Figure 1C).

4. Testing phase

1. During this phase, remove guillotine doors to allow free access to the entire apparatus and place the animal in the middle chamber to let them freely explore the whole apparatus for $15 \mathrm{~min}$.

2. Record their activity during this $15 \mathrm{~min}$.

3. Carry out three post-conditioning tests, i.e., test 1 (T1), test 2 (T2) and test 3 (T3), 2 days, 10 days and 18 days after the last conditioning session in a morphine-free state, respectively (Figure 1A).

4. Vary testing time points for different experimental goals.

NOTE: To minimize the number of rats required, a within-subject design is employed. However, to exclude the extinction effect, a between subject design is required.

\section{A - Pre-conditioning - Conditioning T Test}
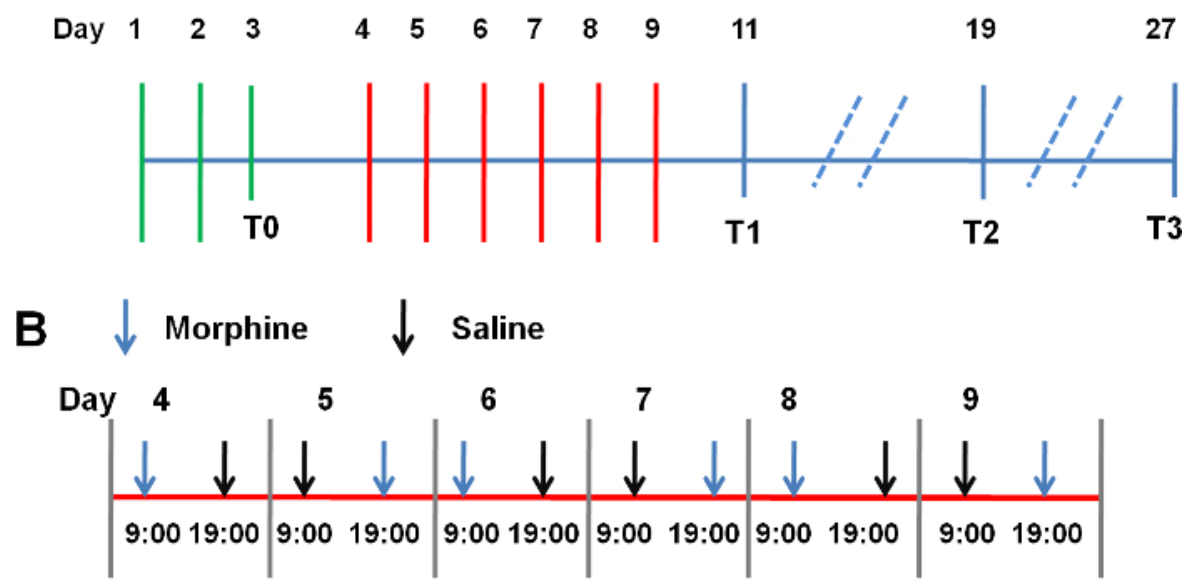

C $\downarrow$ Saline $\quad \downarrow$ Saline

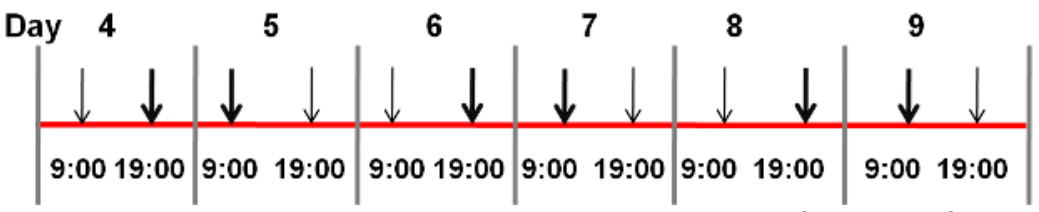

Figure 1: Experimental procedures. (A) Experimental procedures of the whole CPP process. (B) Experimental schedule of the conditioning phase of morphine group. (C) Experimental schedule of the conditioning phase of saline group. Please click here to view a larger version of this figure.

\section{Data Analysis}

1. Calculate the CPP score in morphine(saline) group as: CPP score $=$ the time spent in the drug-paired (non-preferred) chamber/ the total time spent in both conditioning chambers.

2. Perform data analysis of CPP scores and entrances to side chambers with appropriate statistical methods using SPSS.

3. Present all behavioral data as mean \pm SEM, where SEM is the standard error of mean. Consider values of $p<0.05$ as statistically significant and indicate by asterisks $\left(^{*}\right)$ and octothorpesin (\#) in the figures. 


\section{Representative Results}

The following representative results show that a morphine CPP can be successfully established by the protocol described above and long-term morphine reward memory (Figure 2) can be maintained. The number of entries to side chambers, analogous to number of active responses in the self-administration paradigm, reflects the reinforcing property of morphine. The increased number of entries (Figure 3), indicating the incubation of craving, was observed using this morphine CPP paradigm.

\section{CPP memory could last at least 18 days after the last conditioning session.}

Twenty-one rats in the morphine group and 10 rats in the saline group were used to test the expression of the long-term rewarding memory. A mixed ANOVA revealed significant differences in the interaction between group and test $(F(3,87)=4.973, p=0.003)$, test $(F(3,87)=18.237$, $p<0.001)$ and group $(F(1,29)=11.413, p=0.002)$. Significant differences among tests $(F(3,60)=46.628, p<0.001)$ in the morphine group but not saline group $(F(3,27)=1.576, p=0.218)$ were detected by a one-way repeated ANOVA. The multiple comparison analysis with Bonferroni adjustment showed significant differences between the three tests and T0 in the morphine group. As shown in Figure 2, the morphine reward memory lasted at least 18 days and decreased in a time-dependent trend. Data are obtained from a previous published study ${ }^{11}$.

\section{Entrances to side compartments in morphine group increased progressively}

\section{Morphine group}

To test the trend of entrances, group (saline-paired and morphine-paired) as between-subjects factor and test (T0, T1, T2 and T3) as withinsubjects factor were set for a mixed ANOVA. No significant differences in the interaction between group and test $(F(2.311,92.449)=1.915$, $p=0.147)$ and group $(F(1,40)=0.898, p=0.349)$, but significance in test $(F(2.311,92.499)=24.243, p<0.001)$, were detected. The multiple comparison analysis with least significant difference (LSD) test showed significant differences between T1 and T0 ( $p<0.001)$, T2 and T0 ( $p$ $<0.001)$ and T3 and T0 ( < 0.001) in the morphine group in morphine-paired entrances. Concerning saline-paired compartments, statistical significances existed between T2 and T0 $(p=0.002)$ and T3 and T0 $(p=0.003)$ but not T1 and T0 $(p=0.246)$, which is similar to the pattern of incubation of heroin craving ${ }^{24}$. In general, entrances to side chambers in the morphine group increased time-dependently.

Rats entered more frequently to the morphine-paired compartment than saline-paired compartment at T1 $(\mathrm{t}(1,21)=-2.833, \mathrm{p}=0.010)$ and $\mathrm{T} 2$ $(\mathrm{t}(1,21)=-4.458, \mathrm{p}=0.0002)$ but not $\mathrm{T} 3(\mathrm{t}(1,21)=-0.471, \mathrm{p}=0.642)$ (two-tailed paired $\mathrm{t}$-tests).

Saline group

Similar with the morphine group, a mixed ANOVA was applied and revealed no significant differences in the interaction between group and test $(F(3,54)=0.345, p=0.793)$, test $(F(3,54)=1.793, p=0.159)$ and group $(F(1,18)=0.151, p=0.702)$. Thus, entrances to side chambers in the saline group were stable over time.

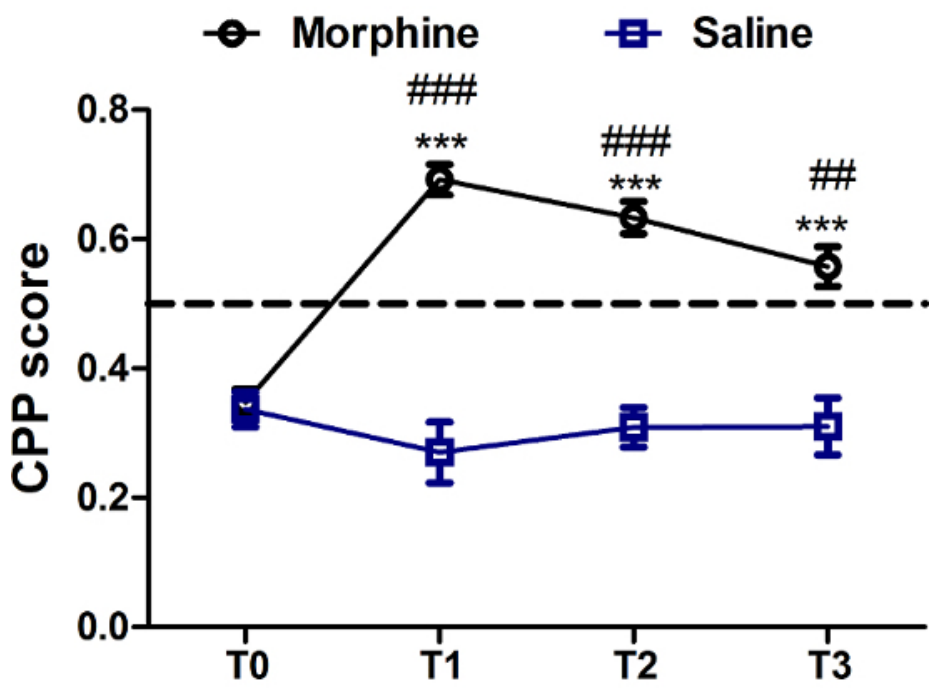

Figure 2: CPP memory can last at least 18 days but declines with time. Memory retention of CPP in the morphine group $(n=21)$ and the saline group $(n=10)$ was assessed by measuring the CPP score for several time points after last conditioning $(T 1,2$ days; T2,10 days and T3, 18 days). All values of CPP scores are mean \pm SEM. \#\#\# indicates $p<0.001$ and \#\# means $p<0.01$ compared with saline group; ${ }^{* * *}$ indicates $p$ $<0.001$ compared with T0 in the morphine group. This figure has been modified from Sun et al.,2017 $7^{11}$. Please click here to view a larger version of this figure. 
A

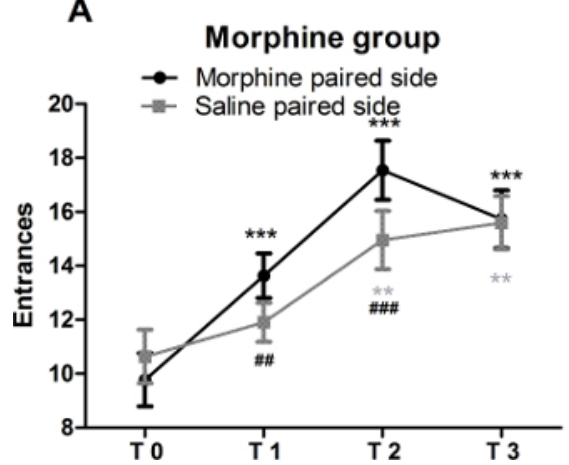

B

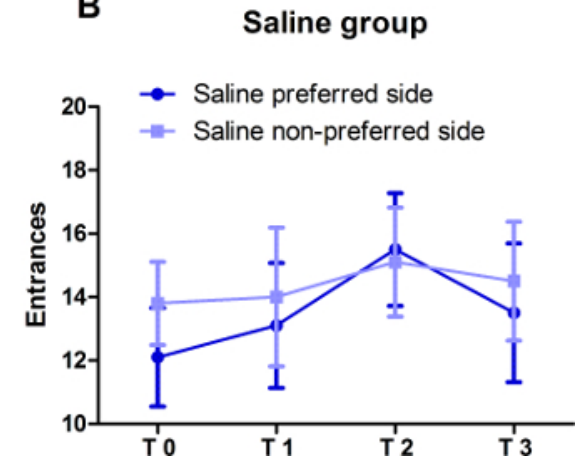

Figure 3: Entrances to side-chambers in morphine and saline groups. Entrances to two side chambers in the morphine group $(n=21)$ and the saline group $(n=10)$ were assessed at several time points after the last conditioning (T1, 2 days; T2,10 days and T3, 18 days $)$ and compared with T0. All values of entrances are mean \pm SEM. In the morphine group $(A)$, entrances to the morphine-paired side increased very significantly from T1 to T3 while entrances of saline-paired chamber only increased significantly from T2. Paired t tests with morphinepaired compartment and saline-paired compartment showed very significant increases in both T1 and T2. In the saline group (B), there are no significant changes in entrances to both sides. This figure has been modified from Sun et al.,2017 ${ }^{11}$. Please click here to view a larger version of this figure.

\section{Discussion}

Here, a method using a morphine CPP paradigm is described to establish long-term reward memory in rat with somatosensory cues as conditioned context. To build such a long-term reward memory, a previous protocol ${ }^{21}$ with a balanced CPP apparatus is modified and the saline and morphine sessions are set at least $10 \mathrm{~h}$ apart. In addition, more conditioning sessions are added during the conditioning phase to enhance the associative learning. Apart from the CPP scores, the entrances to two side chambers are measured and the pattern of increased entrances to side chambers is similar with that of the increased active responses in the self-administration paradigm.

Several factors influence the establishment of the CPP paradigm mentioned above. One key factor is the design of the apparatus: rats in general should have similar preference to both side chambers to ensure the apparatus is balanced. An unbalanced apparatus can mask the acquisition of CPP due to the initial high preference to one side chamber ${ }^{13}$. Therefore, extreme preference to one side chamber (more than $90 \%$ of the total time in any of the two side chambers) should be avoided when choosing the texture of the floor or other contextual cues. For different experimental purposes, experimenters can balance the CPP apparatus by modifying the contextual stimuli. For balanced apparatus, two ways of experimental design are commonly used ${ }^{4}$. One biased way is to pair drugs with the non-preferred chamber with all subjects; an unbiased way is to pair half of the animals with drugs in the non-preferred chamber, the other half with the preferred chamber. Both ways can establish CPP ${ }^{13}$ but studies ${ }^{17,25}$ with the latter design always exclude many subjects due to their strong preference to one side chamber. As individual vulnerability to drug addiction ${ }^{26,27,28}$ exists, a biased design was chosen in this study to avoid exclusion of healthy subjects. One limitation of biased design is the effect of inconsistent preference to side chambers. As shown in Figure 2, rats in the saline group have consistent low CPP scores for the initial non-preferred chamber. Thus, the possibility that inconsistent preference to side chambers could affect the CPP scores in the morphine group is excluded by setting the saline controls. To avoid the ceiling effect of the preferred chamber, we use the initial non-preferred chamber as the morphine paired chamber. To avoid the effect of novelty and stress on the baseline preference to side chambers, three pre-conditioning sessions are performed, and the third session is used as the baseline. The fourth factor is the interval between morphine and saline sessions. In our paradigm, we choose intervals of at least 10 hours to avoid the withdrawal effect in the saline conditioning period. The dosage of morphine is also an important factor. Extremely low doses of morphine, such as $0.01 \mathrm{mg} / \mathrm{kg}^{6}$, did not induce place preference. A dose of $10 \mathrm{mg} / \mathrm{kg}$ induced the highest CPP score ${ }^{17}$ in rats and is commonly used in rat morphine CPP studies ${ }^{17,21,23}$. However, $10 \mathrm{mg} / \mathrm{kg}$ morphine (i.p.) could induce respiratory depression in around $5 \%$ of rats. Lower doses of morphine, such as $5 \mathrm{mg} / \mathrm{kg}$, could be used in future studies. For other species, such as tree shrew, tolerance to drugs should be considered when building the CPP paradigm. Since tree shrew develops fast tolerance to morphine, increased doses of morphine should be used during the conditioning phase ${ }^{29,30}$.

With the modified protocol, a long-term reward memory is established for at least 18 days after 6 days of conditioning ${ }^{11}$ (Figure 2). Future studies should measure whether CPP could be maintained for a longer time. Repeated relapse is the major barrier in treating drug addiction. Stress, environmental cues and drugs could induce relapse. After extinction of the CPP memory, this model could be used to model relapse induced by stress or drugs.

The entrances to the drug-paired chambers in the morphine group increased in the abstinence period ${ }^{11}$ (Figure 3), which is similar with the increased active responses in the self-administration paradigm. However, entrances to the saline-paired chamber also increased with the CPP paradigm in both rats and tree shrews ${ }^{11}$, which is different from the inactive responses of the self-administration paradigm. This difference might due to the different underlying principles of the two paradigms: one is classical conditioning, the other is operant conditioning. Besides, tree shrews with food CPP experiences did not show such a pattern of increased entrances ${ }^{30}$. Therefore, the increased entrances to both side chambers in morphine-experienced animals are thought to represent incubated craving of drugs. Although the limitation of using repeated measures in one subject cannot be excluded, the pattern of increased entries was conserved in rats ${ }^{11}$, tree shrews ${ }^{11,30}$ and monkeys ${ }^{31}$. Nonetheless, a between subject design during the abstinence period is highly recommended for future studies to exclude the extinction effect of repeated measurements.

Apart from the increased entrances, CPP scores with low doses of morphine $(1 \mathrm{mg} / \mathrm{kg}$ and $3 \mathrm{mg} / \mathrm{kg}$ ) increased progressively during the abstinence period $^{17}$. These results suggest that the CPP paradigm could also model the incubation of craving phenomenon. However, an increased CPP score might also represent an increased memory for the conditioned stimulus. In this aspect, entrances to the side chambers 
in a three-chamber apparatus, which are similar to active responses in the self-administration paradigm, more closely resemble the search for drugs or the rewarding effect of drugs. It would be interesting to check the trends of entrances to side chambers during the abstinence period in rat CPP paradigms of lower doses of morphine. Future studies with other drugs and mice should be done to verify whether this phenomenon is conserved across drugs of abuse/species.

\section{Disclosures}

The authors have nothing to disclose.

\section{Acknowledgements}

This work was supported by National Natural Science Foundation of China (NSFC)31741058 (Y.Z.), Shenzhen Governmental grants (JCYJ20170818162613877 and JCYJ20170818163217196) and China Postdoctoral Science Foundation Grant 2017M622826 (K.Z.).

\section{References}

1. Leshner, A. I. Addiction is a brain disease, and it matters. Science. 278 (5335), 45-47 (1997).

2. Koob, G. F., \& Volkow, N. D. Neurocircuitry of Addiction. Neuropsychopharmacology. 35 (1), 217-238 (2010).

3. Zhu, Y., Wienecke, C. F., Nachtrab, G., \& Chen, X. A thalamic input to the nucleus accumbens mediates opiate dependence. Nature. 530 (7589), 219-222 (2016).

4. Tzschentke, T. M. Measuring reward with the conditioned place preference paradigm: a comprehensive review of drug effects, recent progress and new issues. Progress in Neurobiology. 56 (6), 613-672 (1998).

5. Tzschentke, T. M. Measuring reward with the conditioned place preference (CPP) paradigm: update of the last decade. Addiction Biology. 12 (3-4), 227-462 (2007).

6. Mucha, R. F., van der Kooy, D., O'Shaughnessy, M., \& Bucenieks, P. Drug reinforcement studied by the use of place conditioning in rat. Brain Research. 243 (1), 91-105 (1982).

7. van Ree, J. M., Gerrits, M. A., \& Vanderschuren, L. J. Opioids, reward and addiction: An encounter of biology, psychology, and medicine Pharmacological Reviews. 51 (2), 341-396 (1999).

8. Le Merrer, J., Becker, J. A., Befort, K., \& Kieffer, B. L. Reward processing by the opioid system in the brain. Physiological Reviews. 89 (4), 1379-1412 (2009).

9. Bardo, M. T., \& Bevins, R. A. Conditioned place preference: what does it add to our preclinical understanding of drug reward? Psychopharmacology (Berl). 153 (1), 31-43 (2000).

10. Bardo, M. T., Rowlett, J. K., \& Harris, M. J. Conditioned place preference using opiate and stimulant drugs: a meta-analysis. Neuroscience \& Biobehavioral Reviews. 19 (1), 39-51 (1995).

11. Sun, Y., Pan, Z., \& Ma, Y. Increased entrances to side compartments indicate incubation of craving in morphine-induced rat and tree shrew CPP models. Pharmacology Biochemistry and Behavior. 159 62-68 (2017).

12. Sun, Y. M. et al. The caudal part of the posterior insula of rats participates in the maintenance but not the acquisition of morphine conditioned place preference. CNS Neuroscience \& Therapeutics. (2018).

13. Cunningham, C. L., Ferree, N. K., \& Howard, M. A. Apparatus bias and place conditioning with ethanol in mice. Psychopharmacology (Berl). 170 (4), 409-422 (2003).

14. Carles, S.-S., \& Rainer, S. REVIEW: Behavioural assessment of drug reinforcement and addictive features in rodents: an overview. Addiction Biology. 11 (1), 2-38 (2006)

15. Grimm, J. W., Hope, B. T., Wise, R. A., \& Shaham, Y. Neuroadaptation. Incubation of cocaine craving after withdrawal. Nature. 412 (6843), 141-142 (2001).

16. Gawin, F., \& Kleber, H. Pharmacologic treatments of cocaine abuse. Psychiatric Clinics of North America. 9 (3), $573-583$ (1986)

17. Li, Y. Q. et al. Central amygdala extracellular signal-regulated kinase signaling pathway is critical to incubation of opiate craving. $J$ Neurosci. 28 (49), 13248-13257 (2008).

18. Lubbers, B. R. et al. The Extracellular Matrix Protein Brevican Limits Time-Dependent Enhancement of Cocaine Conditioned Place Preference. Neuropsychopharmacology. 41 (7), 1907-1916 (2016).

19. Bardo, M. T., Miller, J. S., \& Neisewander, J. L. Conditioned place preference with morphine: the effect of extinction training on the reinforcing CR. Pharmacology Biochemistry and Behavior. 21 (4), 545-549 (1984).

20. Sun, Y., Zong, W., Zhou, M., Ma, Y., \& Wang, J. Pre-conditioned place preference treatment of chloral hydrate interrupts the rewarding effect of morphine. Pharmacology Biochemistry and Behavior. 135 60-63 (2015).

21. Lin, X., Wang, Q., Cheng, Y., Ji, J., \& Yu, L. C. Changes of protein expression profiles in the amygdala during the process of morphineinduced conditioned place preference in rats. Behavioural Brain Research. 221 (1), 197-206 (2011).

22. Lv, X.-F., Xu, Y., Han, J.-S., \& Cui, C.-L. Expression of activity-regulated cytoskeleton-associated protein (Arc/Arg3.1) in the nucleus accumbens is critical for the acquisition, expression and reinstatement of morphine-induced conditioned place preference. Behavioural Brain Research. 223 (1), 182-191 (2011).

23. Meng, Z., Liu, C., Hu, X., \& Ma, Y. Somatosensory cortices are required for the acquisition of morphine-induced conditioned place preference. PLoS One. 4 (11), e7742 (2009).

24. Shalev, U., Morales, M., Hope, B., Yap, J., \& Shaham, Y. Time-dependent changes in extinction behavior and stress-induced reinstatement of drug seeking following withdrawal from heroin in rats. Psychopharmacology (Berl). 156 (1), 98-107 (2001).

25. Zhao, Y. et al. Electrolytic lesions of the bilateral ventrolateral orbital cortex inhibit methamphetamine-associated contextual memory formation in rats. Brain Research. (2015).

26. Vanderschuren, L. J., \& Everitt, B. J. Drug seeking becomes compulsive after prolonged cocaine self-administration. Science. $\mathbf{3 0 5}$ (5686), 1017-1019 (2004).

27. Deroche-Gamonet, V., Belin, D., \& Piazza, P. V. Evidence for addiction-like behavior in the rat. Science. 305 (5686), 1014-1017 (2004), 
28. Augier, E. et al. A molecular mechanism for choosing alcohol over an alternative reward. Science. 360 (6395), 1321-1326 (2018).

29. Sun, Y. M., Yang, J. Z., Sun, H. Y., Ma, Y. Y., \& Wang, J. H. Establishment of tree shrew chronic morphine dependent model. Dongwuxue Yanjiu. 33 (1), 14-18 (2012).

30. Duan, Y., Shen, F., Gu, T., \& Sui, N. Addiction: From Context-Induced Hedonia to Appetite, Based on Transition of Micro-behaviors in Morphine Abstinent Tree Shrews. Frontiers in Psychology. 7816 (2016).

31. Wu, X. et al. Morphine-induced conditioned place preference in rhesus monkeys: Resistance to inactivation of insula and extinction. Neurobiology of Learning and Memory. 131 192-200 (2016). 